\title{
IMPACTOS AMBIENTAIS DE CAVA DE MINERAÇÃO DE CALCÁRIO A PARTIR DE ANAGLIFOS E GEOPROCESSAMENTO
}

\author{
João Carlos Valério Vieira de Albuquerque ${ }^{1}$, Arthur Costa Falcão Tavares ${ }^{2}$ \\ 'Discente do Centro de Ciências Agrárias, Universidade Federal de Alagoas, BR 104, Km 85, s/n, Rio Largo - AL, CEP 57.100-000. \\ joao.albuquerque@ceca.ufal.br. \\ ${ }^{2}$ Docente do Centro de Ciências Agrárias, Universidade Federal de Alagoas, BR 104, Km 85, s/n, Rio Largo - AL, CEP 57.100-000. \\ acftavar@ceca.ufal.br
}

\begin{abstract}
RESUMO: As pesquisas para visualização 3D através da estereoscopia, como meio de simulação da realidade, ainda desafiam as atuais tecnologias em grau e qualidade da imersão alcançada. Assim, este trabalho propôs manipular fotografias aéreas para gerar anaglifos afim de detectar atividade mineradora e seu processo de urbanização, além de projetar seus impactos ambientais. Os materiais utilizados foram fotos aéreas oriundas de levantamentos aerofotogramétricos realizados em 1940, 1962, 1972, 1978 e 2010 além de softwares específicos para criar anaglifo e georreferenciar anaglifos, além de detectar e calcular as áreas de cava. A análise dos anaglifos multitemporais indicou uma mudança drástica no uso do solo da área da cava de mineração. Em 1972 e 1978 a projeção de impacto ambiental foi respectivamente classes alta e muito alta, 0 que comprometeria seriamente a continuidade dos trabalhos de lavra pelas distâncias dos locais das explosões às áreas habitadas, dadas as restrições relacionadas aos valores da Carga Máxima de Explosivo (CME) a ser detonada. Consideração que o guia para avaliação dos efeitos provocados pelo uso de explosivos nas minerações em áreas urbanas (NBR 9653), foi criado em 1986, as classes de projeção para os anos de 1940 e 1962 podem ter chegado até muito alta.
\end{abstract}

Palavras-chave: impacto ambiental, mineração de calcário, uso do solo, visualização 3D, fotografia aérea

\section{ENVIRONMENTAL IMPACTS OF LIMESTONE MINING USING ANAGLYPHS AND GEOPROCESSING}

\begin{abstract}
Research for 3D visualization through stereoscopy, as a reality simulating, still challenges current technologies in degree and quality of achieved immersion. Thus, this propose proposed to manipulate aerial photographs to generate anaglyphs in order to detect mining activity and its urbanization process, in addition to prognoses its environmental impacts. The materials used were aerial photos from aerophotogrammetric surveys accomplished in 1940, 1962, 1972, 1978 and 2010, as well as specific software to create and georeferencing anaglyphs, such as to detect and calculate mining pit. The analysis of the multitemporal anaglyphs indicated a drastic change in the land use of the mining pit area. In 1972 and 1978 the prognoses of environmental impact was respectively high and very high classes, which would seriously jeopardize the continuity of the mining works by the distances of the explosions to inhabited areas, due to restrictions determined by values of Maximum Load of Explosive (CME) to be detonated. Considering the guide for assessing the effects of the use of explosives on mining in urban areas (NBR 9653) was created in 1986, the prognoses for the years 1940 and 1962 may have reached very high classes.
\end{abstract}

Keywords: environmental impact, limestone mining, landuse, 3d visualization, aerial photo

\section{INTRODUÇÃO}

A Organização de Pesquisa Científica da Indústria (CSIRO) está pesquisando o uso da visualização $3 \mathrm{D}$ envolvendo o desenvolvimento de técnicas para integrar complexos conjuntos de dados diferentes, multitemporais e 3D usando software e tecnologias para a entrega e segurança das informações (FOWLE, 2003).

A veracidade da estereoscopia como meio de simulação ainda desafia as atuais tecnologias de Realidade Virtual em termos do grau e qualidade da imersão alcançada e da congruência dos estímulos 
visuais e cinestésicos. A aplicação de processos digitais à fotografia estereoscópica pode resultar em novos modos estéticos de apresentar e representar a paisagem como uma experiência abrangente e que tais apresentações podem ser usadas para investigar a maneira pela qual a aura de um espaço paisagístico é estabelecida e mantida (WALCH, 1998).

Historicamente a utilização de fotografias aéreas tem proporcionado informações precisas e confiáveis nos estudos de levantamentos de uso e cobertura vegetal da terra. Neste contexto, os produtos usuais das fotos aéreas ainda são capazes de fornecer melhores alternativas quanto à resolução espacial, temporal e possibilidade de análise estereoscópica (SIRTOLI, 1998).

Concomitantemente, $\quad 0$ constante desenvolvimento de sofwares e hardwares tem favorecido o desenvolvimento de técnicas para obtenção de informações mais precisas dos dados registrados nas imagens. No entanto, para cada aplicação é necessário o desenvolvimento de um processamento metodológico específico (SIRTOLI, 1998; BALIEIRO et al., 2015).

Sabe-se que qualquer atividade mineradora durante sua operação, e mesmo depois de desativada, pode trazer problemas ao seu entorno. Como o seu reconhecimento no campo é impossível visto que a pedreira se transformou numa quadra urbanizada com edificações residenciais, os levantamentos aerofotogramétricos antigos e transformados em anaglifos aparecem como sendo uma promissora ferramenta para diagnosticar possíveis causas de impactos negativos de antigas áreas mineradas.

Os danos causados pela exploração mineral no QF se intensificaram com o decorrer dos anos, transformando e prejudicando cada vez mais o meio ambiente. Isso, não apenas nas áreas mineradas, mas também nas áreas vizinhas, sendo necessária a implantação de programas que monitorem e quantifiquem a área alterada e degradada pela mineração, e visem minimizar os impactos ambientais e socioeconômicos causados por esta atividade no meio ambiente e nas comunidades ao qual estão envolvidas (VON AHN; SIMON, 2017; DINIZ et al., 2014).

Um conjunto de fotos aéreas históricas foi obtido em formato digital para detecção de pedreira de calcário desativada no município de Piracicaba/ SP. Os voos ocorreram em 1940, 1962, 1972 e 1978 capturando fotos aéreas em preto e branco com diferentes escalas de 1:20000, 1:25000 e 1:35.000. As fotos aéreas históricas, anaglifos e mapas auxiliares foram usados e processados em um Sistema de Informação Geográfica (SIG). (EMIL, 2010).

0 objetivo deste trabalho foi manipular fotografias aéreas para obtenção de anaglifos a fim de detectar atividade mineradora e projetar os impactos ambientais causados por esta atividade em diferentes épocas.

\section{MATERIAL E MÉTODOS}

Os materiais básicos utilizados neste trabalho foram imagens aéreas multitemporais, em formato digital, oriundas do acervo de levantamentos aerofotogramétricos de 1940, 1962, 1972 e 1978 pertencentes ao Prof. Arthur Tavares (CECA/UFAL) e oriundas do levantamento aerofotogramétrico de 2010 (EMPLASA, 2018).

Para o processamento e análise das fotografias aéreas multitemporais foram utilizados os seguintes softwares:

- Stereo Photo Maker: trata-se também de um software gratuito aqui utilizado para criação do anaglifo a partir do par de fotografias aéreas.

- QGIS (versão 3.4.1): trata-se de um software livre e gratuito aqui utilizado para o Georreferenciamento das fotografias aéreas e dos anaglifos, além da criação dos arquivos shapefiles e medição das áreas com presença e ausência de cava de mineração.

- Libreofice (versão 6.1.3): trata-se de um software livre e gratuito aqui utilizado para avaliação e projeção do impacto da atividade mineradora no seu entorno.

Os arquivos shapefile criados a partir dos anaglifos, tiveram no uso de óculos específico para visualização tridimensional, um suporte fundamental. Os anaglifos por sua vez tiveram papel primordial na detecção e confirmação da presença da cava de mineração, visto que os pares de fotografias aéreas datam de 1940, 1962, 1972 e 1978, o que inviabiliza verificar a informação em campo.

Para o desenvolvimento metodológico do presente trabalho foram realizadas as etapas do fluxograma abaixo (Figura 1). 
Figura 1. Fluxograma com as etapas metodológicas realizadas no trabalho.

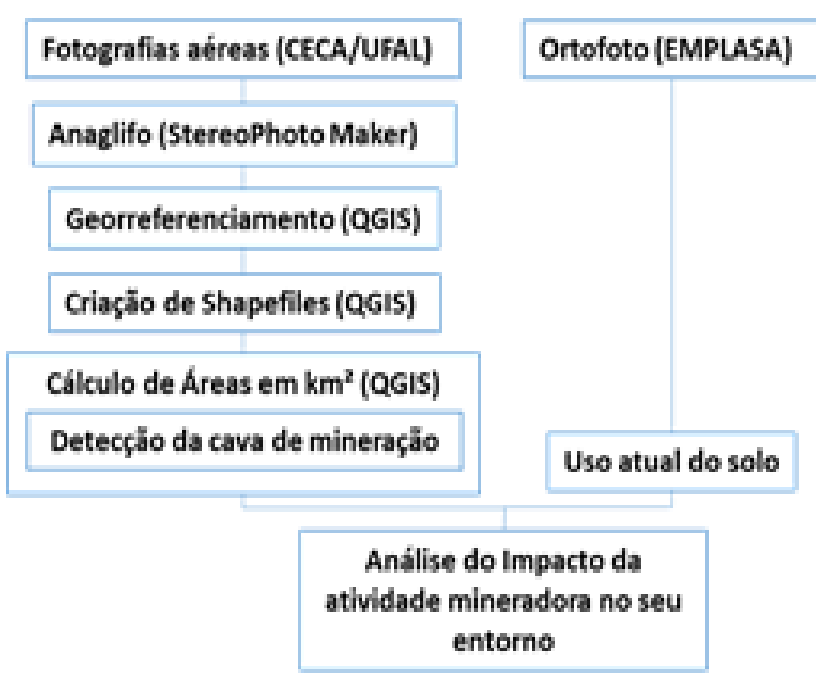

\section{RESULTADOS E DISCUSSÃO}

Para o estudo foi selecionada uma área do município de Piracicaba, Estado de São Paulo, a fim de detectar área com cava de mineração (Figura 2).

Figura 2. Localização da área deste estudo.

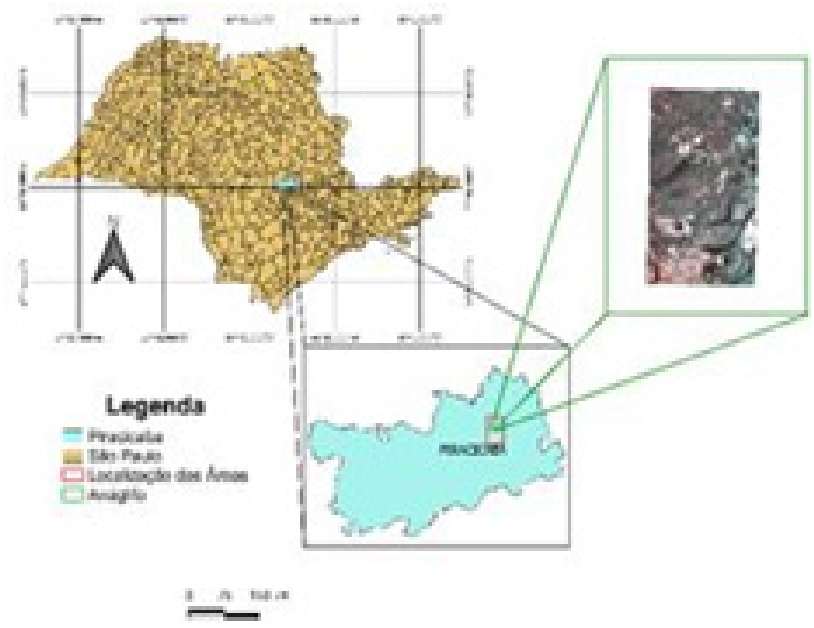

A análise dos anaglifos (1940, 1962, 1972 e 1978), permitiu detectar a presença e a expansão da cava de mineração. 0 georreferenciamento dos anaglifos permitiu a criação dos arquivos shapefiles e como resultado da fotointerpretação realizada no anaglifo do ano de 1962, por exemplo, foi delimitada (azul), detectada e calculada a área da cava de mineração (Figura 3).
Figura 3. Fotointerpretação do anaglifo para criação de shapefile e cálculo da área de cava de mineração (1962).

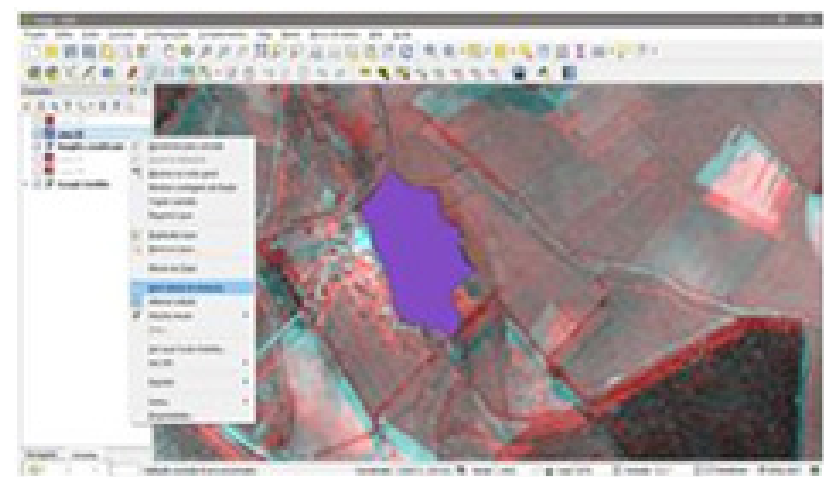

A ortofoto de 2010 (EMPLASA, 2018), indicou o desaparecimento da cava de mineração e o surgimento de uma área totalmente urbanizada (Figura 4). Tal área foi identificada sobrepondo-se 0 arquivo $\mathrm{kml}$, originado do shapefile (vermelho) da cava de mineração em 1978, o qual apresentou a maior expansão da área, à ortofoto de 2010.

Figura 4. Fotointerpretação da foto aérea de 2010 a partir do shapefile de 1978.

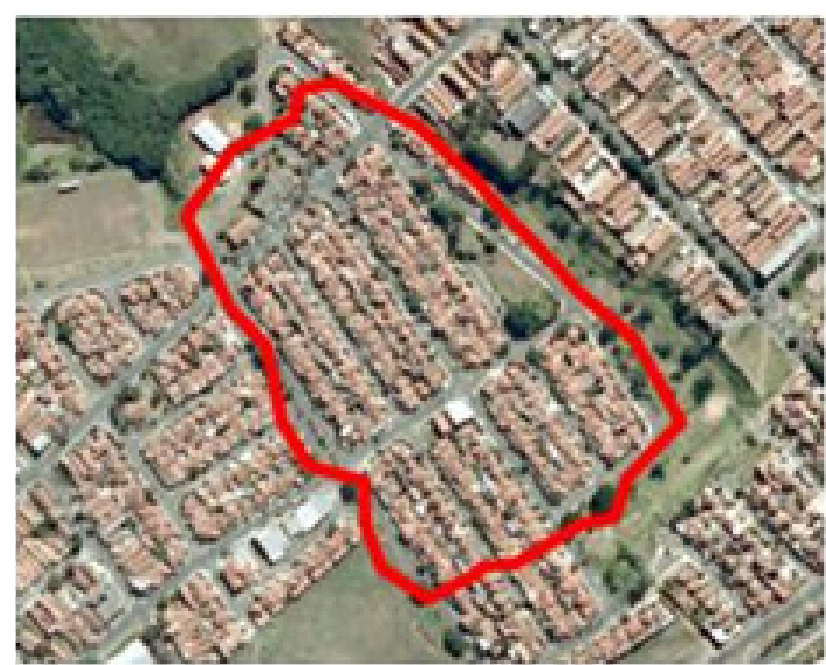

Os resultados indicaram uma mudança drástica no uso do solo da área estudada (Tabela 1). Considerando apenas o período entre 1940 e 1978 a área da cava de mineração teve sua maior variação entre os anos de 1940 e 1962 (73\%) cujos valores aumentaram de 16053 para $27808 \mathrm{~m}^{2}$. A maior variação da distância da cava à área urbana mais próxima ocorreu entre 1962 e 1972 (66 \%). Em 2010 os valores de área diminuíram para 0 tanto para área quanto para distância, fazendo com que as variações 
caíssem também em $100 \%$, visto que toda a área da cava estava urbanizada. Vale destacar a inversão dos valores de área e de distância entre 1940 e 1978, ou seja, 0 aumento dos valores de área para cada ano.

Tabela 1. Valores da área da cava, da distância da cava para área urbana, e das respectivas variações entre 1940 e de 2010.

\begin{tabular}{ccccc}
\hline Ano & Área $\left(\mathrm{m}^{2}\right)^{(1)}$ & $\%^{(2)}$ & Distância $(\mathrm{m})^{(3)}$ & $\%{ }^{(4)}$ \\
\hline 1940 & 16053 & 0 & 1620 & 0 \\
1962 & 27808 & 73 & 1180 & 27 \\
1972 & 42470 & 53 & 400 & 66 \\
1978 & 68434 & 61 & 200 & 50 \\
2010 & 0 & 100 & 0 & 100 \\
\hline
\end{tabular}

(1): Área da cava de mineração;

(2): Variação da área da cava de mineração;

(3): Distância entre a cava de mineração e a área urbana mais próxima;

(4): Variação da distância entre a cava de mineração e a área urbana mais próxima

A avaliação e projeção do impacto ambiental se baseou na curva de Dallora Neto, (2004) obtida pela equação de máxima energia correlacionando as cargas máximas de explosivos passíveis de serem detonadas instantaneamente com a distância entre o local da detonação e o local a ser preservado. No caso específico do local enfocado, em 1972 e 1978 a projeção de impacto foi classificada como alta e muito alta respectivamente. Para estes anos, os parâmetros estabelecidos pela curva comprometeriam seriamente a continuidade dos trabalhos de lavra pelas distâncias (400m em 1972 e 200m em 1978) às áreas habitadas, dadas as restrições dela decorrentes em relação aos valores da CME (35kg em 1972 e 5 kg em 1978). Para classificação como baixo e médio, respectivamente para os anos de 140 e 1962, foram projetados valores de CME (141kg em 1940 e 103kg em 1962) observando a tendência da curva (Tabela 2).

Tabela 2. Valores da distância da cava para área urbana mais próxima e da CME, e as respectivas classes de projeção de impacto ambiental, entre 1940 e 2010.

\begin{tabular}{cccc}
\hline Ano & Distância $(\mathbf{m})^{(1)}$ & CME $(\mathbf{k g})^{(2)}$ & Projeção $^{(3)}$ \\
\hline 1940 & 1620 & $141^{(4)}$ & Baixa \\
1962 & 1180 & $103^{(5)}$ & Média \\
1972 & 400 & 35 & Alta \\
1978 & 200 & 5 & Muito alta \\
2010 & 0 & 0 & (6) \\
\hline
\end{tabular}

(1): Distância entre a cava de mineração e a área urbana mais próxima;

(2): Carga máxima de explosivo a ser detonada instantaneamente;

(3): Projeção de impacto ambiental;

(4): Valor projetado em planilha eletrônica utilizando a fórmula =(D4*F5)/D5;

(5): Valor projetado em planilha eletrônica utilizando a fórmula $=\left(\mathrm{D} 5^{\star} \mathrm{F} 6\right) / \mathrm{D} 6$;

(6): sem projeção.

\section{CONCLUSÕES}

A projeção de impacto ambiental pela presença da cava de mineração aumentou enormemente entre 1940 e 1978. Correlacionando os valores de distância da cava até a área urbana e de CME percebeu-se que em um período de 48 anos a classe considerada baixa em 1940 saltou para muito alta em 1978.

Considerando o fato do Guia para avaliação dos efeitos provocados pelo uso de explosivos nas minerações em áreas urbanas (NBR 9653) ser uma 
norma criada em 1986, muito antes dos períodos aqui analisados, as classificações de projeção de impacto ambiental para os anos de 1940 e 1962 poderiam ter sido média, alta ou até muito alta. Mesmo a cava estando há distâncias seguras é provável que os valores de CME utilizados nestas épocas não estivessem de acordo com as normas e legislações específicas mais atuais.

\section{AGRADECIMENTOS}

Agradecemos ao Prof. Arthur Tavares (CECA) UFAL) por disponibilizar as fotos aéreas de 1940, 1962, 1972 e 1978 do acervo de dados utilizados no seu doutorado.

\section{REFERÊNCIAS}

BALIEIRO, C. P. P.; GARCIA, E.; SANTOS, L. B.; MALLMANN, G. M. Uso de imagens de alta resolução espacial na detecção de áreas de mineração na Amazônia brasileira. In: SIMPÓSIO BRASILEIRO DE SENSORIAMENTO REMOTO, 17. (SBSR), 2015, João Pessoa. Anais... São José dos Campos: INPE, 2015. p. 6397-6404.

DALLORA NETO, C. Análise das vibrações resultantes do desmonte de rocha em mineração de calcário e argilito posicionada junto à área urbana de Limeira (SP). 82 p. Dissertação (mestrado em Geociências e Meio Ambiente), Universidade Estadual Paulista, SP, 2004.

DINIZ, J. M. F. S.; DOS REIS, A. A.; ACERBI JUNIOR, F. W.; GOMIDE, L. R. Detecção da expansão da área minerada no quadrilátero ferrífero, Minas Gerais, no período de 1985 a 2011 através de técnicas de sensoriamento remoto. Boletim de Ciências Geodésicas, 2014. 20, 683-700.
EMIL, M. K. Land degradation assessment for an abandoned coal mine with geospatial information technologies. $113 \mathrm{p}$. Thesis (Master of Science in Mining Engineering Department), Middle East Technical University, 2010.

EMPLASA - Empresa Paulista de Planejamento Metropolitano. Mapa. Disponível em: < http://www. mapeiasp.sp.gov.br/Mapa >. Acesso em: 1 de novembro de 2018.

FOWLE, K. G. E. The use of computer graphics and visualisation (from reconstruction to training) for the resource sector of western Australia. 239 p. Thesis (Doctor of Philosophy in Department of Chemical and Environmental Engineering), University of Nottingham, 2003.

SIRTOLI, A. E. Detecção da expansão de área de mineração através de técnicas de sensoriamento remoto. 98 p. Dissertação (Mestrado em Ciências Florestais), Universidade Federal do Paraná, PR, 1998.

VON AHN, M. M; SIMON, A. L. H. Geomorphological mapping and geodiversity: study at the minas do camaquã geosite protection area (Brazil). Revista Brasileira de Geomorfologia, 2017, 18, 427-442.

WALCH, M. An analysis of experiential space at the close of the twentieth century. $52 \mathrm{p}$. Thesis (Master Fine Art by Research), MFA, University of Tasmania, School of Art. 1998. 